\title{
HESS J1818-154, a new composite supernova remnant discovered in TeV gamma rays and $\mathrm{X}$-rays
}

H.E.S.S. Collaboration, A. Abramowski ${ }^{1}$, F. Aharonian ${ }^{2,3,4}$, F. Ait Benkhali ${ }^{2}$, A. G. Akhperjanian ${ }^{5,4}$, E. Angüner ${ }^{6}$, G. Anton ${ }^{7}$, S. Balenderan ${ }^{8}$, A. Balzer ${ }^{9}, 10$, A. Barnacka ${ }^{11}$, Y. Becherini ${ }^{12}$, J. Becker Tjus ${ }^{13}$, K. Bernlöhr ${ }^{2,6}$, E. Birsin ${ }^{6}$, E. Bissaldi ${ }^{14}$, J. Biteau ${ }^{15}$, M. Böttcher ${ }^{16}$, C. Boisson ${ }^{17}$, J. Bolmont $^{18}$, P. Bordas $^{19}$, J. Brucker ${ }^{7}$, F. Brun ${ }^{2}$, P. Brun ${ }^{20}$, T. Bulik ${ }^{21}$, S. Carrigan ${ }^{2}$, S. Casanova ${ }^{16,2}$, M. Cerruti ${ }^{17,22}$, P. M. Chadwick ${ }^{8}$, R. Chalme-Calvet ${ }^{18}$, R. C. G. Chaves ${ }^{20}$, A. Cheesebrough ${ }^{8}$, M. Chrétien ${ }^{18}$, S. Colafrancesco ${ }^{23}$, G. Cologna $^{24}$, J. Conrad $^{25,26}$, C. Couturier ${ }^{18}$, Y. Cui ${ }^{19}$, M. Dalton ${ }^{27,28}$, M. K. Daniel ${ }^{8}$, I. D. Davids ${ }^{16,29}$, B. Degrange ${ }^{15}$, C. Deil ${ }^{2}$, P. deWilt ${ }^{30}$, H. J. Dickinson ${ }^{25}$, A. Djannati-Atai ${ }^{31}$, W. Domainko ${ }^{2}$, L. O'C. Drury ${ }^{3}$, G. Dubus ${ }^{32}$, K. Dutson ${ }^{33}$, J. Dyks ${ }^{11}$, M. Dyrda ${ }^{34}$, T. Edwards ${ }^{2}$, K. Egberts ${ }^{14}$, P. Eger $^{2}$, P. Espigat ${ }^{31}$, C. Farnier ${ }^{25}$, S. Fegan ${ }^{15}$, F. Feinstein ${ }^{35}$, M. V. Fernandes ${ }^{1}$, D. Fernandez ${ }^{35}$, A. Fiasson ${ }^{36}$, G. Fontaine ${ }^{15}$, A. Förster ${ }^{2}$, M. Füßling ${ }^{10}$, M. Gajdus ${ }^{6}$, Y. A. Gallant ${ }^{35}$, T. Garrigoux ${ }^{18}$, G. Giavitto ${ }^{9}$, B. Giebels ${ }^{15}$, J. F. Glicenstein ${ }^{20}$, M.-H. Grondin ${ }^{2,24}$, M. Grudzińska ${ }^{21}$, S. Häffner ${ }^{7}$, J. Hahn² ${ }^{2}$ J. Harris ${ }^{8}$, G. Heinzelmann ${ }^{1}$, G. Henri ${ }^{32}$, G. Hermann ${ }^{2}$, O. Hervet ${ }^{17}$, A. Hillert ${ }^{2}$, J. A. Hinton ${ }^{33}$, W. Hofmann ${ }^{2}$, P. Hofverberg ${ }^{2}$, M. Holler ${ }^{10}$, D. Horns ${ }^{1}$, A. Jacholkowska ${ }^{18}$, C. Jahn ${ }^{7}$, M. Jamrozy ${ }^{37}$, M. Janiak ${ }^{11}$, F. Jankowsky ${ }^{24}$, I. Jung ${ }^{7}$, M. A. Kastendieck ${ }^{1}$, K. Katarzyński ${ }^{38}$, U. Katz ${ }^{7}$, S. Kaufmann ${ }^{24}$, B. Khélifï ${ }^{31}$, M. Kieffer ${ }^{18}$, S. Klepser ${ }^{9}$, D. Klochkov ${ }^{19}$, W. Kluźniak ${ }^{11}$, T. Kneiske ${ }^{1}$, D. Kolitzus ${ }^{14}$, Nu. Komin ${ }^{36}$, K. Kosack ${ }^{20}$, S. Krakau ${ }^{13}$, F. Krayzel ${ }^{36}$, P. P. Krüger ${ }^{16,2}$, H. Laffon ${ }^{27}$, G. Lamanna ${ }^{36}$, J. Lefaucheur ${ }^{31}$, A. Lemière ${ }^{31}$, M. Lemoine-Goumard ${ }^{27}$, J.-P. Lenain ${ }^{18}$,

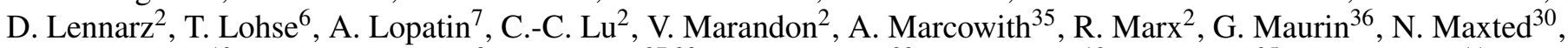
M. Mayer ${ }^{10}$, T. J. L. McComb ${ }^{8}$, J. Méhault ${ }^{27,28}$, P. J. Meintjes ${ }^{39}$, U. Menzler ${ }^{13}$, M. Meyer ${ }^{25}$, R. Moderski ${ }^{11}$,

M. Mohamed ${ }^{24}$, E. Moulin ${ }^{20}$, T. Murach ${ }^{6}$, C. L. Naumann ${ }^{18}$, M. de Naurois ${ }^{15}$, J. Niemiec ${ }^{34}$, S. J. Nolan ${ }^{8}$, L. Oakes ${ }^{6}$, S. Ohm ${ }^{33}$, E. de Oña Wilhelmi ${ }^{2}$, B. Opitz ${ }^{1}$, M. Ostrowski ${ }^{37}$, I. Oya ${ }^{6}$, M. Panter ${ }^{2}$, R. D. Parsons ${ }^{2}$, M. Paz Arribas ${ }^{6}$, N. W. Pekeur ${ }^{16}$, G. Pelletier ${ }^{32}$, J. Perez ${ }^{14}$, P.-O. Petrucci ${ }^{32}$, B. Peyaud ${ }^{20}$, S. Pita ${ }^{31}$, H. Poon ${ }^{2}$, G. Pühlhofer ${ }^{19}$,

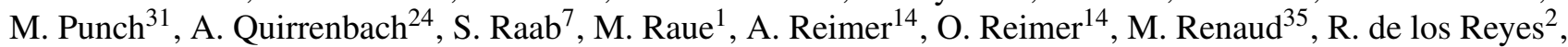
F. Rieger ${ }^{2}$, L. Rob ${ }^{40}$, C. Romoli ${ }^{3}$, S. Rosier-Lees ${ }^{36}$, G. Rowell ${ }^{30}$, B. Rudak ${ }^{11}$, C. B. Rulten ${ }^{17}$, V. Sahakian ${ }^{5,4}$, D. A. Sanchez ${ }^{2,36}$, A. Santangelo ${ }^{19}$, R. Schlickeiser ${ }^{13}$, F. Schüssler ${ }^{20}$, A. Schulz ${ }^{9}$, U. Schwanke ${ }^{6}$, S. Schwarzburg ${ }^{19}$, S. Schwemmer ${ }^{24}$, H. Sol ${ }^{17}$, G. Spengler ${ }^{6}$, F. Spies ${ }^{1}$, Ł. Stawarz $^{37}$, R. Steenkamp ${ }^{29}$, C. Stegmann ${ }^{10,9}$, F. Stinzing ${ }^{7}$, K. Stycz ${ }^{9}$, I. Sushch ${ }^{6,16}$, A. Szostek ${ }^{37}$, J.-P. Tavernet ${ }^{18}$, T. Tavernier ${ }^{31}$, A. M. Taylor ${ }^{3}$, R. Terrier ${ }^{31}$, M. Tluczykont ${ }^{1}$, C. Trichard ${ }^{36}$, K. Valerius ${ }^{7}$, C. van Eldik ${ }^{7}$, B. van Soelen ${ }^{39}$, G. Vasileiadis ${ }^{35}$, C. Venter ${ }^{16}$, A. Viana ${ }^{2}$, P. Vincent ${ }^{18}$, H. J. Völk ${ }^{2}$, F. Volpe ${ }^{2}$, M. Vorster ${ }^{16}$, T. Vuillaume ${ }^{32}$, S. J. Wagner ${ }^{24}$, P. Wagner ${ }^{6}$, M. Ward ${ }^{8}$, M. Weidinger ${ }^{13}$, Q. Weitzel ${ }^{2}$, R. White ${ }^{33}$, A. Wierzcholska ${ }^{37}$, P. Willmann ${ }^{7}$, A. Wörnlein ${ }^{7}$, D. Wouters ${ }^{20}$, V. Zabalza ${ }^{2}$, M. Zacharias ${ }^{13}$, A. Zajczyk ${ }^{11,35}$, A. A. Zdziarski ${ }^{11}$, A. Zech ${ }^{17}$, and H.-S. Zechlin ${ }^{1}$

(Affiliations can be found after the references)

Received 25 October 2013 / Accepted 9 December 2013

\section{ABSTRACT}

\begin{abstract}
Composite supernova remnants (SNRs) constitute a small subclass of the remnants of massive stellar explosions where non-thermal radiation is observed from both the expanding shell-like shock front and from a pulsar wind nebula (PWN) located inside of the SNR. These systems represent a unique evolutionary phase of SNRs where observations in the radio, X-ray, and $\gamma$-ray regimes allow the study of the co-evolution of both these energetic phenomena. In this article, we report results from observations of the shell-type SNR G 15.4+0.1 performed with the High Energy Stereoscopic System (H.E.S.S.) and XMM-Newton. A compact TeV $\gamma$-ray source, HESS J1818-154, located in the center and contained within the shell of G $15.4+0.1$ is detected by H.E.S.S. and featurs a spectrum best represented by a power-law model with a spectral index of $-2.3 \pm 0.3_{\text {stat }} \pm 0.2_{\text {sys }}$ and an integral flux of $F(>0.42 \mathrm{TeV})=\left(0.9 \pm 0.3_{\text {stat }} \pm 0.2_{\text {sys }}\right) \times 10^{-12} \mathrm{~cm}^{-2} \mathrm{~s}^{-1}$. Furthermore, a recent observation with $X M M$-Newton reveals extended X-ray emission strongly peaked in the center of $\mathrm{G} 15.4+0.1$. The X-ray source shows indications of an energydependent morphology featuring a compact core at energies above $4 \mathrm{keV}$ and more extended emission that fills the entire region within the SNR at lower energies. Together, the X-ray and VHE $\gamma$-ray emission provide strong evidence of a PWN located inside the shell of G $15.4+0.1$ and this SNR can therefore be classified as a composite based on these observations. The radio, X-ray, and $\gamma$-ray emission from the PWN is compatible with a one-zone leptonic model that requires a low average magnetic field inside the emission region. An unambiguous counterpart to the putative pulsar, which is thought to power the PWN, has been detected neither in radio nor in X-ray observations of G 15.4+0.1.
\end{abstract}

Key words. X-rays: individuals: G15.4+0.1 - gamma rays: general - methods: observational - supernovae: individual: HESS J1818-154 $\mathrm{X}$-rays: general 


\section{Introduction}

In the aftermath of supernova explosions, a rich variety of highly energetic non-thermal processes can be observed. At the shock front of the expanding supernova remnant (SNR) shell where the ejecta from the explosion interacts with the surrounding interstellar matter, charged particles can be accelerated up to energies of $\mathrm{TeV}$, and possibly $\mathrm{PeV}$, through the standard diffusive shock mechanism (DSA; for a review, see Baring 1997) and can give rise to intense broad-band electromagnetic radiation. The non-thermal radiation is produced either by energetic hadrons through production and subsequent decay of neutral pions, or by leptons that radiate through synchrotron and inverse Compton (IC) emission.

The supernova explosion can also leave behind a rapidly rotating neutron star that constitutes a second major source of non-thermal emission. This can take the form of pulsed emission from the pulsar's magnetosphere and/or of an extended pulsar wind nebula (PWN) powered by a relativistic particle outflow from the central engine (for a comprehensive review see Gaensler \& Slane 2006).

The particle wind from the pulsar is decelerated by the inertia of the higher density outer medium, and a termination shock is formed. In the wind, particles are advected outward toward the termination shock, following the frozen-in magnetic field lines in an orderly flow, resulting in a (synchrotron) under-luminous feature close to the pulsar. At the shock front, the particles are isotropized and accelerated, and subsequently produce both synchrotron and IC radiation when gyrating in the nebular magnetic field in the downstream flow.

Typical broad-band spectral energy distributions (SEDs) of PWNe appear to be dominated by leptonic processes and show two broad peaks. The first one extends from radio to X-ray energies arising from synchrotron radiation, whereas the second one is peaked in the $\gamma$-ray regime that arises from IC up-scattering of ambient low-energy photon fields.

$\mathrm{X}$-ray observations of PWNe with high angular resolution instruments, such as XMM-Newton, are particularly useful since they may differentiate between the extended synchrotron emission from the PWN, seen downstream of the termination shock, and the magnetospheric non-thermal emission from the pulsar itself (for a review of recent results obtained in X-rays, see Uchiyama 2012). By comparing the properties of these two components, one can learn about the physical processes responsible for the efficient particle acceleration mechanism energizing PWNe. Very-high-energy (VHE, $E>100 \mathrm{GeV}$ ) $\gamma$-ray observations of PWNe are complementary to X-ray observations since they trace particles with a longer lifetime, through an emission mechanism largely independent of the nebular magnetic field, unlike the synchrotron X-ray emission.

$\mathrm{X}$-ray and VHE $\gamma$-ray observations have established two classes of PWNe based on their observational properties. In young systems, such as the Crab nebula, the pulsar is still close to its birthplace at the center of the SNR and surrounded by an $\mathrm{X}$-ray and a VHE $\gamma$-ray nebula, and the latter is normally unresolved owing to its small size and the relatively poor angular resolution of the ground-based instruments. In middle aged systems, such as HESS J1303-631 (Abramowski et al. 2012) or HESS J1825-137 (Aharonian et al. 2006c), the IC nebula is found to be significantly extended and offset from the pulsar, in contrast to the much smaller and still pulsar-centered $\mathrm{X}$-ray nebulae. This difference in size is generally attributed to the much shorter cooling timescale of the highest energy lepton population that produces X-ray synchrotron radiation, compared to the lower energy leptons giving rise to the IC component. Additionally, for these older systems, the interaction between the expanding PWN and the reverse shock of the SNR likely leads to a compression of the PWN followed by a re-expansion phase. If the SNR evolves into an inhomogeneous interstellar medium (ISM), the impact of the reverse shock on the PWN evolution may vary within the nebula, which may lead to the asymmetric and offset emission regions that are particularly visible in the IC regime. For a detailed study of evolved PWNe in SNRs, see Blondin et al. (2001).

A rare subtype of SNRs/PWNe include so-called composite SNRs (Helfand \& Becker 1987), which show distinct evidence of both a PWN and a SNR shell (like G 0.9+0.1 and G 21.5-0.9, see e.g., Aharonian et al. 2005; Matheson \& Safi-Harb 2005). These systems are excellent laboratories for investigating the co-evolution of PWNe and SNRs and, particularly, the potential interaction between the two. To explore the general nature of particle acceleration, transport, and energy-loss mechanisms in such environments, it is necessary to increase the number of well-studied systems in various stages of evolution. For this purpose, detailed multiwavelength studies have to be performed to define the properties and environment of the SNR, as well as to probe the full extent of the non-thermal spectral energy distribution of the PWN.

The SNR G $15.4+0.1$ is a poorly studied object that was initially discovered in a $90 \mathrm{~cm}$ survey of the inner Galaxy conducted by the Very Large Array (VLA; Brogan et al. 2006). The SNR was reported to have a shell-like morphology with a size of about $14^{\prime} \times 15^{\prime}$ and an average spectral index $\alpha=-0.6 \pm 0.2$, indicating that the radio emission is dominated by non-thermal synchrotron emission from the shell. A recent study by Castelletti et al. (2013) places the SNR at a distance of $(4.8 \pm 1.0) \mathrm{kpc}$ and discovered a molecular cloud coincident with the SNR. For the reasons outlined above, this source is a potential emitter of both $\mathrm{X}$-rays and VHE $\gamma$ rays. Interestingly, this SNR is slightly larger than the H.E.S.S. point-spread-function (PSF; $\sim 6^{\prime}$ ), which allows a morphological comparison of the VHE $\gamma$-ray emission with the size of the SNR shell.

In this paper the nature of the newly detected VHE $\gamma$-ray source HESS J1818-154 coincident with SNR G 15.4+0.1 is investigated, based on results obtained with H.E.S.S., as well as from a recent X-ray observation performed with XMM-Newton. The morphology and spectral energy distribution are discussed in the context of a composite SNR scenario.

\section{H.E.S.S. observations and results}

\subsection{The H.E.S.S. telescope array}

The High Energy Stereoscopic System (H.E.S.S. Aharonian et al. 2006a) is an array of imaging atmospheric Cherenkov telescopes for VHE $\gamma$-ray astronomy that detects Cherenkov light emitted from $\gamma$-ray- induced air showers. H.E.S.S. is comprised of four identical $12 \mathrm{~m}$ diameter telescopes in a square of $120 \mathrm{~m}$ and is located on the southern hemisphere in the Khomas highland of Namibia (latitude $23^{\circ} 16^{\prime} 17^{\prime \prime} S$ ) at a height of $1800 \mathrm{~m}$ above sea level. Employing a stereoscopic technique for detecting air showers, an angular resolution better than $0.1^{\circ}$, and an energy resolution of $15 \%$ are achieved, coupled with a high background rejection power. Its unprecedented sensitivity to $\gamma$ rays permits H.E.S.S. to detect a point source with a flux of $1 \%$ of the Crab nebula at a significance of $5 \sigma$ in about $25 \mathrm{~h}$ of observations. In 2012, the H.E.S.S. observatory was extended with a fifth $\sim 28 \mathrm{~m}$ diameter telescope at the center of the array - H.E.S.S. II, 
which will improve the sensitivity of the instrument, particularly in the energy regime above a few tens of $\mathrm{GeV}$.

\subsection{Data and analysis methods}

The region around G 15.4+0.1 was observed with H.E.S.S. between 2004 and 2011; the dataset consists primarily of observations from the H.E.S.S. Galactic Plane Survey (Aharonian et al. 2006b) and offset observations of nearby sources. The dataset in this region has a live time of $\sim 120 \mathrm{~h}$ after standard H.E.S.S. quality selection (Aharonian et al. 2006a), although the effective live time is considerably lower $(\sim 80 \mathrm{~h})$ because of the large average offset of the pointings from the target $\left(1.9^{\circ}\right)$. The data was taken in a series of runs with typical durations of $28 \mathrm{~min}$ at a mean zenith angle of $23^{\circ}$.

The data set was analyzed using the Hillas second moment method (Hillas 1985) for distinguishing between $\gamma$-rayand hadron-induced extensive air showers (EAS). Hard cuts (Aharonian et al. 2006a) were used, where at least 200 photoelectrons are required in each recorded EAS image. These cuts provide improved angular resolution ${ }^{1}$ which is crucial for this particular analysis, as well as an increased average energy threshold of $420 \mathrm{GeV}$. To generate $2 \mathrm{D}$ images, the adaptive ring background method (Carrigan et al. 2013) was used, which is a similar method to the traditional ring background method (Berge et al. 2007) but with the modification that the ring is allowed to grow according to the requirements in the field-ofview (FoV), and is therefore suitable when analyzing crowded regions. A minimal inner radius of $0.6^{\circ}$ and a ring thickness of $0.3^{\circ}$ were used. The size of the on-region was $0.1^{\circ}$. For the spectral analysis, the background was estimated using the reflected region method (Berge et al. 2007), where the background is derived from circular off-source regions with the same angular size $\left(0.15^{\circ}\right)$ and camera offset as the on-source region, located at the best-fit position of the source in question ${ }^{2}$. This technique therefore minimizes systematic errors that might be introduced from an incomplete knowledge of the radial acceptance. The statistical significances for both the images and the spectral analysis were derived from the number of off-source (background) and on-source events, following the likelihood ratio procedure in $\mathrm{Li}$ \& Ma (1983). All presented H.E.S.S. results have been crosschecked with an alternative analysis chain using an independent calibration and $\gamma$-ray/hadron separation method (de Naurois \& Rolland 2009), which give results that are consistent within statistical errors.

\subsection{Results}

Figure 1 shows a map of the acceptance-corrected and smoothed $\gamma$-ray excess in the region around the SNR G 15.4+0.1. A source of $\gamma$ rays from the direction of the SNR is clearly visible and detected with a significance of $8.2 \sigma$. Fitting the data with a 2D Gaussian model convolved with the PSF of the instrument results in a best-fit position of the source of $\alpha=18^{\mathrm{h}} 18^{\mathrm{m}} 4^{\mathrm{s}} .8 \pm 3^{\mathrm{s}} .1_{\text {stat }}$ and $\delta=-15^{\circ} 28^{\prime} 1^{\prime \prime} \pm 43^{\prime \prime}{ }_{\text {stat }}{ }^{3}\left(l \sim 15.41^{\circ}, b \sim 0.16^{\circ}\right)$, and the source is thus assigned the identifier HESS J1818-154. In addition to the statistical uncertainty of this fit, there is also a systematic uncertainty due to the pointing precision of the telescope array of about 20" (Gillessen et al. 2005). The morphology of the

\footnotetext{
1 The $68 \%$ containment radius of the PSF is $0.076^{\circ}$.

2 The size of the on-source region was selected to have a source containment of roughly $90 \%$.

3 All coordinates in this work are given in $\mathbf{J} 2000.0$ format.
}

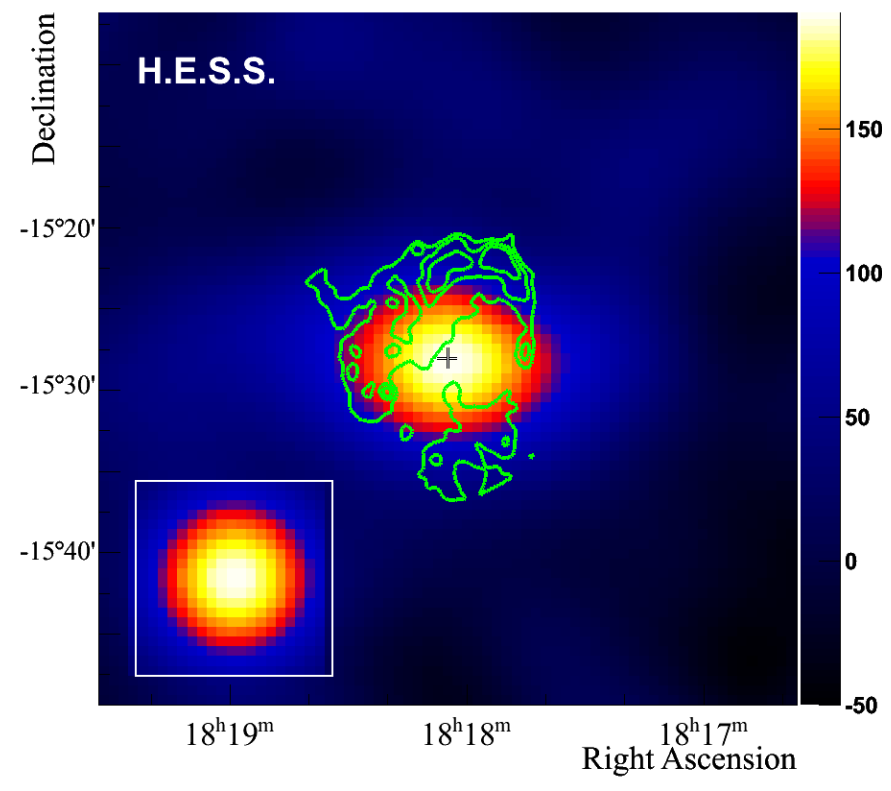

Fig. 1. VHE $\gamma$-ray excess around the SNR G 15.4+0.1. The image has been corrected for the varying exposure across the FoV and has been smoothed with a $2 \mathrm{D}$ Gaussian with a width of $0.06^{\circ}$. The H.E.S.S. PSF for this analysis is shown in the bottom left inset. The color scale is chosen such that the blue-red transition occurs at roughly $4 \sigma$ significance. The best-fit centroid of the $\gamma$-ray excess is indicated by a cross, the size of which corresponds to the sum of both statistical and systematic uncertainties. The green contours show the intensity (at $0.0175,0.035$ and $0.0525 \mathrm{Jy} \mathrm{beam}^{-1}$ ) of the radio emission from the SNR shell, based on 90-cm VLA observations (Brogan et al. 2006).

$\mathrm{TeV}$ emission is compatible with its originating in a point-like source. The $99 \%$ confidence upper limit on the Gaussian width of the source is $0.072^{\circ}$. No significant ellipticity of the source was found.

A spectral analysis was performed and yielded 117 excess $\gamma$-ray counts in the spectral extraction region. The resulting differential spectrum is shown in Fig. 2 and is well fitted in the energy range $0.42 \mathrm{TeV}-12.0 \mathrm{TeV}$ by a power law,

$$
\begin{aligned}
\frac{\mathrm{d} N}{\mathrm{~d} E}= & \left(0.9 \pm 0.2_{\text {stat }} \pm 0.2_{\text {sys }}\right) \\
& \times 10^{-13}\left(\frac{E}{E_{\mathrm{dec}}}\right)^{-2.3 \pm 0.3_{\text {stat }} \pm 0.2_{\text {sys }}} \mathrm{cm}^{-2} \mathrm{~s}^{-1} \mathrm{TeV}^{-1}
\end{aligned}
$$

where $E_{\mathrm{dec}}=1.9 \mathrm{TeV}$ is the decorrelation energy. This corresponds to an integral flux $F(>0.42 \mathrm{TeV})=\left(0.9 \pm 0.3_{\text {stat }} \pm 0.2_{\text {sys }}\right) \times$ $10^{-12} \mathrm{~cm}^{-2} \mathrm{~s}^{-1}$.

\subsection{Origin of the TeV emission}

Since the size of the shell of $\mathrm{G} 15.4+0.1$ in radio is comparable to the size of the H.E.S.S. PSF, it is not obvious whether the observed $\mathrm{TeV}$ emission originates in the shell of the SNR, in an unknown source located inside the shell, or from a combination of both. Indeed, simulations show that a pure shell-type emission from G 15.4+0.1 convolved with the H.E.S.S. PSF results in a center-dominated source. A simulation study was therefore performed to investigate whether a shell-like origin of the $\mathrm{TeV}$ emission can be excluded based on the observed extension of HESS J1818-154.

$\mathrm{TeV}$ emission was simulated from two source components: a point-like and a shell-like source, the former positioned at the center of the latter. The morphology of the shell-type source was 


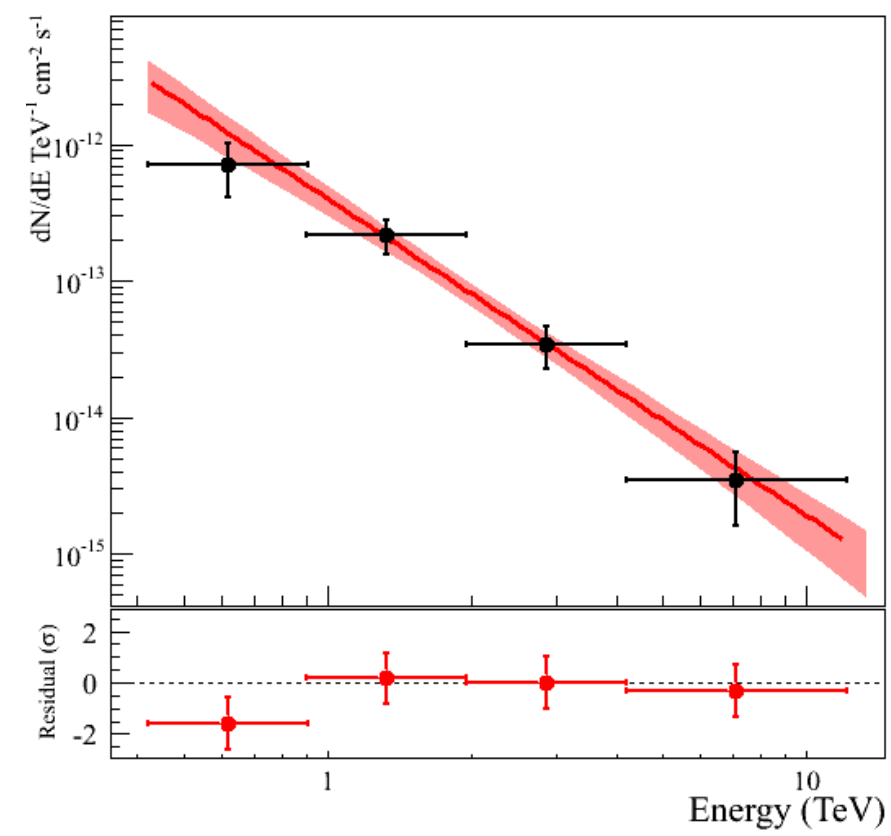

Fig. 2. Differential VHE $\gamma$-ray spectrum of HESS J1818-154. The error bars represent $1 \sigma$ statistical errors. The result of a power-law fit to the spectral data points is also shown, together with the $1 \sigma$ error band. The residuals between the power-law fit and the datapoints are shown in the bottom plot.

obtained by fitting a 2D projection of an elliptical 3D model of a thin shell to the SNR G 15.4+0.1, as seen in $90 \mathrm{~cm}$ radio data (see Sect. 4), and yielded a size of roughly $12^{\prime} \times 18^{\prime}$. The simulations were done using the same exposure and background maps as in the real observations of HESS J1818-154, and the total simulated flux was set to match the observed flux. The relative contribution of the shell component to the total flux was varied from $10 \%$ to $100 \%$ in steps of $10 \%$. For each set, 500 observations were simulated, and the width of the combined emission was derived for each simulation by fitting a 2D Gaussian model. The mean and standard deviation for each distribution of the fitted Gaussian width were then derived and are plotted in Fig. 3. This can be compared to the best-fit extension of HESS J1818-154 $\left(0.03^{\circ}\right)$ which is indicated. This extension is not statistically significant but is nonetheless used in this particular study since it results in a more conservative result. From the observed differences in size, a scenario where a pure uniform shell-type emission is reconstructed with the Gaussian size of HESS J1818-154 can be excluded at a significance of $6.4 \sigma$. Furthermore, a shell-type contribution above $45 \%$ is excluded at $3 \sigma$. Models of non-uniform $\mathrm{TeV}$ shell emission have not been investigated, but considering that the H.E.S.S. source is located roughly at the center of the SNR, such scenarios could probably be rejected with higher confidence than above.

\section{XMM-Newton data analysis and results}

To explore a PWN scenario for the newly detected compact VHE $\gamma$-ray source HESS J1818-154, the H.E.S.S. collaboration successfully proposed to observe the region with XMM-Newton to search for point-like and diffuse X-ray counterparts. In this section, the analysis of the European Photon Imaging Camera (EPIC) MOS (Turner et al. 2001) and PN (Strüder et al. 2001) data from this observation (Obs. ID: 0691390101, PI: Hofverberg) is presented. The data were analyzed with the

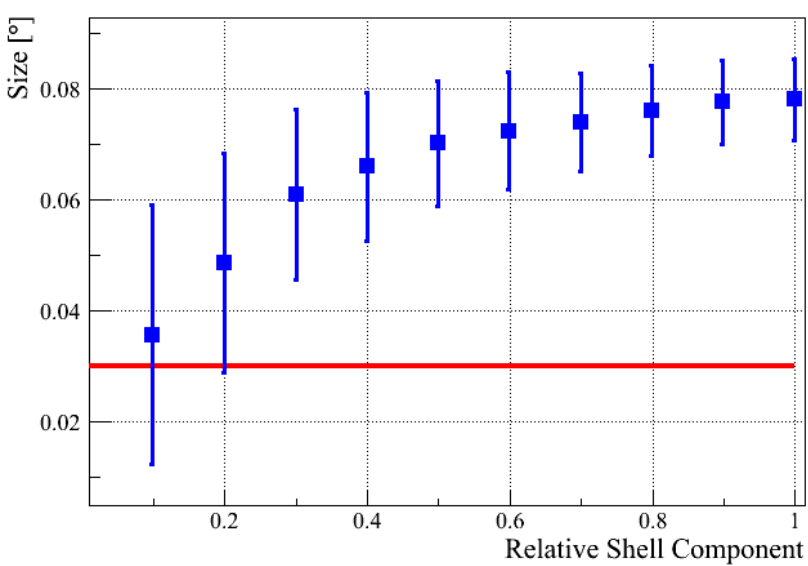

Fig. 3. Reconstructed Gaussian size for the combined $\mathrm{TeV}$ emission from a point-like and a shell-type source (blue datapoints). The $x$-axis indicates the relative contribution from the shell component to the total flux. The red line indicates the non-significant, best-fit extension of HESS J1818-154.

XMM-Newton Science Analysis System (SAS) version 12.0.1, supported by tools from the FTOOLS package and XSPEC version 12.5.0 (Arnaud 1996) for spectral modeling. Some tools from the CIAO 4.4 software package were used for image processing.

This observation was affected by some intervals of strong background flaring activity. To clean the data, a good timeinterval (GTI) screening was employed, based on the full FoV 7-15 keV light curve provided by the standard processing chain. Using thresholds of $8 \mathrm{cts} / \mathrm{s}$ for PN and $3 \mathrm{cts} / \mathrm{s}$ for MOS, the resulting net (total) exposures are $23 \mathrm{ks}(30 \mathrm{ks})$ for PN and $30 \mathrm{ks}$ $(32 \mathrm{ks})$ for MOS. For all spectra and images, good single and multiple events were selected: $($ FLAG $=0)$, PATTERN $\leq 4(\mathrm{PN})$, and PATTERN $\leq 12(\mathrm{MOS})$.

\subsection{Point-like $X$-ray sources}

To detect X-ray point sources, the SAS standard maximumlikelihood technique for source detection was used in several energy bands: $0.2-0.5 \mathrm{keV}, 0.5-1.0 \mathrm{keV}, 1.0-2.0 \mathrm{keV}, 2.0-$ $4.5 \mathrm{keV}, 4.5-10.0 \mathrm{keV}$, and $0.5-10.0 \mathrm{keV}$. After merging the source lists from the three cameras and the individual energy bands, 75 unique point sources were detected in this observation. The observed flux of the faintest detected point source in the list is $\sim 4 \times 10^{-14} \mathrm{erg} \mathrm{cm}^{-2} \mathrm{~s}^{-1}(0.5-10.0 \mathrm{keV}$, assuming a power-law spectrum with photon index -2 ), which is considered to be close to the actual detection limit considering the large sample size of 75 sources.

Because the focus of this work is to search for potential counterparts to HESS J1818-154, only the five point sources that are within a region corresponding to the location and potential size of HESS J1818-154 are discussed below. These sources are labeled in Fig. 4, which shows an exposure-corrected and colorcoded image created from EPIC-PN counts maps (see figure caption for details of the energy bands). Fortunately, the count statistics for all of these sources were sufficient to perform a spectral analysis. To extract source counts, a circular region was used with the $99 \%$ containment radius of the PSF at the respective detector area, centered on the position given by the source detection algorithm. For each source, the background spectrum was extracted from a nearby source-free region on the same detector CCD. Spectra from the three EPIC cameras were fitted in 
Table 1. X-ray point sources in the direction of HESS J1818-154.

\begin{tabular}{|c|c|c|c|c|c|c|c|c|c|}
\hline No. & RA & Dec & $\begin{array}{l}\text { Name } \\
\text { (XMMU) }\end{array}$ & Counts $^{1}$ & $\begin{array}{l}N_{\mathrm{H}}^{2} \\
\left(10^{22} \mathrm{~cm}^{-2}\right)\end{array}$ & $\begin{array}{l}\Gamma \text { or } k T^{3} \\
(-/ \mathrm{keV})\end{array}$ & $\begin{array}{l}F_{\mathrm{X}}{ }^{4} \\
10^{-14} \mathrm{erg} \mathrm{cm}^{-2} \mathrm{~s}^{-1}\end{array}$ & $p$-value ${ }^{5}$ & Class $^{6}$ \\
\hline 1 & $18^{\mathrm{h}} 17^{\mathrm{m}} 44^{\mathrm{s}}$ & $-15^{\circ} 27^{\prime} 33^{\prime \prime} 7$ & J181744-152733.7 & 1832 & $11_{-2.0}^{+2.7}$ & $\Gamma=-1.8_{+0.3}^{-0.4}$ & & 0.95 & HEXT \\
\hline 2 & $18^{\mathrm{h}} 17^{\mathrm{m}} 46^{\mathrm{s}}$ & $-15^{\circ} 24^{\prime} 18^{\prime \prime} .9$ & J181746-152418.9 & 494 & $0.19_{-0.07}^{+0.27}$ & $k T=0.14_{-0.02}^{+0.02}$ & 0.3 & 0.88 & STAR \\
\hline 3 & $18^{\mathrm{h}} 17^{\mathrm{m}} 48^{\mathrm{s}}$ & $-15^{\circ} 25^{\prime} 57^{\prime \prime} 0$ & $\mathrm{~J} 181748-152557.0$ & 128 & $<1.9$ & $\Gamma=-1.9_{+0.8}^{-0.9}$ & $1.1 \pm 0.6$ & 0.39 & HGAL \\
\hline 4 & $18^{\mathrm{h}} 17^{\mathrm{m}} 55^{\mathrm{s}}$ & $-15^{\circ} 25^{\prime} 59^{\prime} 0$ & $\mathrm{~J} 181755-152559.0$ & 350 & $<0.23$ & $k T=0.21_{-0.07}^{+0.03}$ & $1.3 \pm 0.4$ & 0.59 & STAR \\
\hline 5 & $18^{\mathrm{h}} 18^{\mathrm{m}} 07^{\mathrm{s}}$ & $-15^{\circ} 29^{\prime} 55^{\prime \prime} .0$ & J181807-152955.0 & 152 & $<0.95$ & $\Gamma=-2.5_{+0.7}^{-0.8}$ & $1.1 \pm 0.7$ & 0.85 & HGAL \\
\hline
\end{tabular}

Notes. All quoted uncertainties correspond to the $1 \sigma$ confidence interval; The energy range of the fit is $0.5-10.0 \mathrm{keV}$; ${ }^{(1)}$ number of excess counts summed over all three EPIC cameras; ${ }^{(2)}$ hydrogen column density. The "<"-sign indicates $99 \%$ confidence upper limits; ${ }^{(3)}$ power-law photon index $(\Gamma)$ or black-body temperature $(k T)$, depending on which model yielded the better fit; ${ }^{(4)}$ observed (absorbed) energy flux (0.5-10.0 keV) derived from the best-fit model; ${ }^{(5)}$ fit probability; ${ }^{(6)}$ assigned source class (see text): hard extragalactic (HEXT), hard galactic (HGAL), soft galactic (STAR).

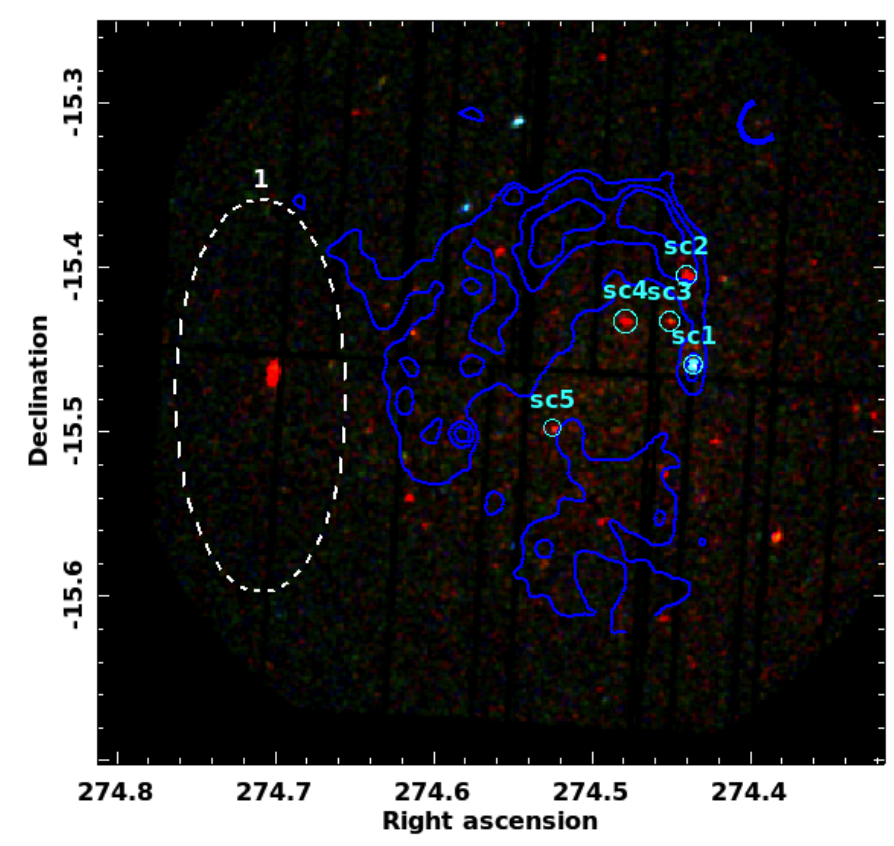

Fig. 4. Exposure-corrected and color-coded EPIC-PN counts image of the region around HESS J1818-154. The energy bands are 0.5$2.0 \mathrm{keV}$ (red), 2.0-4.5 keV (green), and 4.5-10.0 keV (blue). Overplotted are the $90 \mathrm{~cm}$ radio contours of G $15.4+0.1$ (blue). The five detected point sources compatible with the position and possible extent of HESS J1818-154 are marked by numbered circles (cyan). The dashed line (white, labeled " 1 ") shows the region used for the spectral analysis of the background.

parallel. To account for thermal and non-thermal emission, two spectral models were tested to reproduce the spectrum from each source, namely an absorbed power law and an absorbed blackbody model. Table 1 summarizes the results for the five point sources. In each of these cases, one of the models was clearly preferred by a statistical significance of at least $3 \sigma$, and we only show the results for the preferred model. For some sources the fit only provided upper limits for the column density $N_{\mathrm{H}}$.

The results from the spectral analysis were used to classify the point sources to determine their most likely origin. Source 1 is by far the brightest source of the sample, and it features an intrinsically hard spectrum that is strongly absorbed. The derived value for $N_{\mathrm{H}}$ is a factor of $\sim 5$ more than the total Galactic value toward this direction $\left(1.85 \times 10^{22} \mathrm{~cm}^{-2}\right.$, see Dickey \& Lockman 1990). Therefore, this source is most likely of extragalactic origin so was classified as "hard extragalactic (HEXT)". In contrast, sources 2 and 4 exhibit only very low absorption and have soft thermal spectra with temperatures of $0.1-0.2 \mathrm{keV}$. Such spectra are typical of stars featuring coronal activity and/or binary interaction, which can occur during a wide variety of their evolutionary stages (for a recent survey with XMM-Newton, see Nebot Gomez-Moran et al. 2013). Therefore, these two sources were classified as "STAR". The two remaining sources show intrinsically hard spectra, but their upper limits on the column density are either at the level of or below the total Galactic value, which might suggest a Galactic origin, such as cataclysmic variables, X-ray binaries, or pulsars. Sources 3 and 5 are thus classified as "hard galactic (HGAL)".

A search for periodicities in the signal of the five point sources was performed by analyzing the fast Fourier transform (FFT) power spectra derived from light curves in three energy bands: $0.5-2.0 \mathrm{keV}, 2.0-10.0 \mathrm{keV}$, and $0.5-10.0 \mathrm{keV}$. However, no significant peaks were visible above the background noise. The frame times for the full-frame modes used in this XMM-EPIC observation are 2.6 s for MOS and $73.4 \mathrm{~ms}$ for $\mathrm{PN}$, respectively, and thus there is no sensitivity for periodicities below a few times these values.

\subsection{Diffuse $X$-ray emission}

The second part of the XMM-Newton data analysis was dedicated to the search for diffuse X-ray emission spatially coincident with the VHE $\gamma$-ray source HESS J1818-154, which is located in the center of the SNR G 15.4+0.1. The procedure presented here is very similar to previous studies of diffuse X-ray emission from unidentified VHE $\gamma$-ray sources, such as HESS J1626-490 (Eger et al. 2011) and HESS J1747-248 (Eger et al. 2010).

In a first step, events around all detected X-ray point sources were removed from the EPIC event lists to facilitate analysis of only diffuse emission. For each source, a circular exclusion region was defined, centered on the position given by the SAS source detection algorithm, with a radius equivalent to the 99\% containment radius of the PSF at the respective position in the focal plane. To estimate the non-X-ray background (NXB) caused by readout noise, charged particles passing the detector, etc., filter-wheel-closed datasets were used that are provided by the XMM-Newton background working group (Carter \& Read 2007). The same GTI-filtering criteria as for the source observation were also applied to the NXB datasets. 

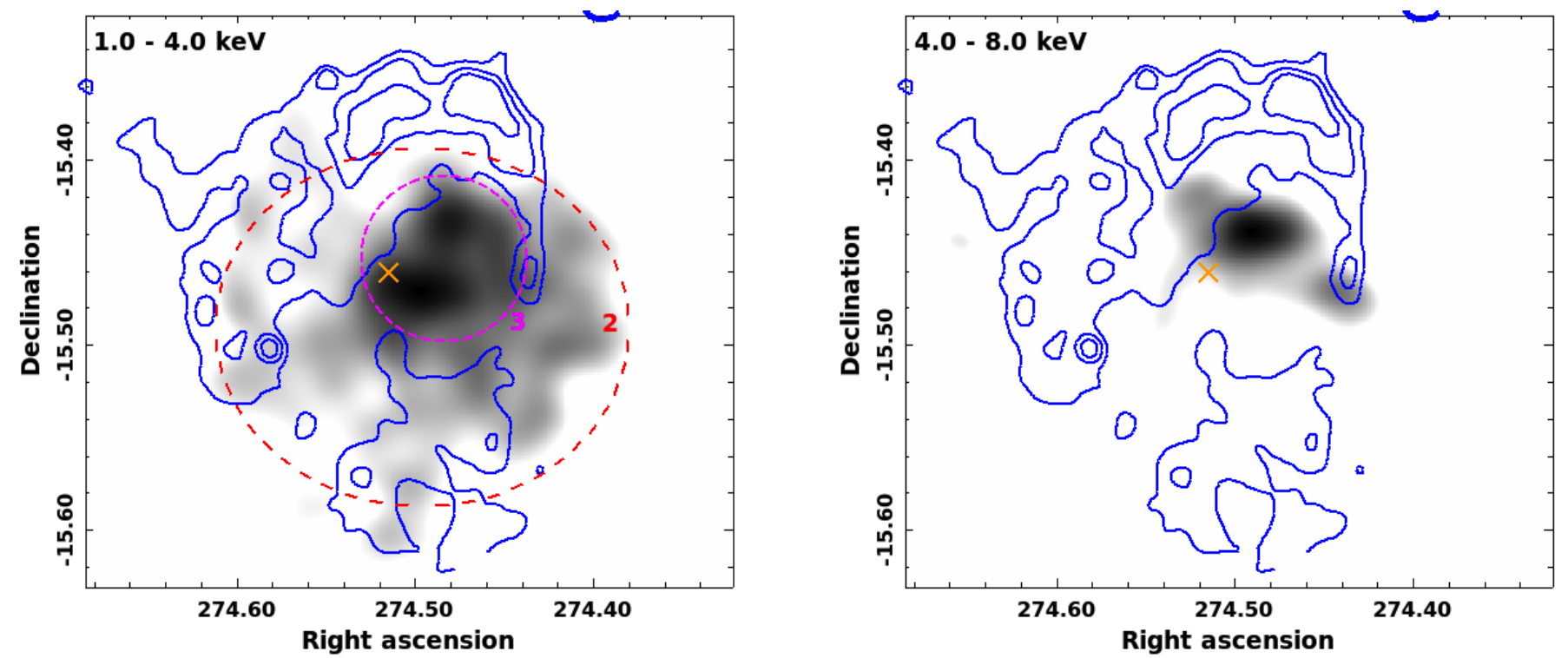

Fig. 5. Smoothed (Gaussian kernel with $0.01^{\circ}$ sigma), exposure-corrected images of diffuse X-ray emission in two energy bands (as indicated in the figures). The color scale is linear between the threshold, as defined in the text, and the maximum pixel intensity. The $90 \mathrm{~cm}$ radio contours of G 15.4+0.1 are overplotted (blue). The dashed lines indicate the regions used for spectral analysis. The cross (orange) indicates the best-fit position of HESS J1818-154.

\subsubsection{Maps of diffuse X-ray emission}

To create maps of diffuse X-ray emission, images of X-ray counts were extracted in the two energy bands $1.0-4.0 \mathrm{keV}$ and $4.0-8.0 \mathrm{keV}$. These bands were chosen to avoid the strong astrophysical background at energies below $\sim 1 \mathrm{keV}$ and a large part of the NXB that is dominant above 8-9 keV. The holes in the maps resulting from the removal of point sources were refilled using the CIAO tool dmfilth where the Poisson count statistics from annular regions around each source are used to create fake event distributions inside the excluded regions. Maps extracted from the NXB dataset in the same energy bands were subtracted from the source maps. To account for differences in exposure and in the mean level of the background count rate, the NXB maps were rescaled based on the ratio of highenergy events (10-12 keV) between source and NXB data before subtraction (a method discussed in detail by Majerowicz et al. 2002). Finally, the NXB-subtracted maps were smoothed using a Gaussian kernel with a size of $0.01^{\circ}$ and divided by the exposure maps smoothed in the same manner. In a last step the maps from all three EPIC instruments were added for a combined image.

These maps should only contain a large-scale astrophysical background component stemming from thermal emission of hot gas in the Galactic plane and potential excess emission connected to the SNR and/or the putative PWN in its center. The level of the diffuse astrophysical component present in these maps was estimated by the mean $\left(m_{\mathrm{AB}}\right)$ and standard deviation $\left(\sigma_{\mathrm{AB}}\right)$ of pixel intensities from regions outside the SNR shell, using an exclusion region centered on the SNR (RA: $18^{\mathrm{h}} 18^{\mathrm{m}} 03^{\mathrm{s}}$, Dec: $\left.-15^{\circ} 27^{\prime} 58^{\prime \prime}\right)$ with a radius of 8.5. The lower threshold (white) of the maps' color scales was set to $m_{\mathrm{AB}}+\sigma_{\mathrm{AB}}$ to significantly suppress the contribution from the astrophysical background. Figure 5 shows the resulting maps for both energy bands. As suggested by these images, there is clear excess above the astrophysical background component centered inside the SNR radio shell. Furthermore, this excess emission appears to be more compact at higher energies. Unfortunately, owing to the low surface brightness in the outer regions of the diffuse emission, its true extent is hard to quantify with the available data.

\subsubsection{Spectra of the diffuse excess emission}

The large extent and relatively low surface brightness of the diffuse excess emission makes a spectral analysis challenging, particularly concerning accurate estimation of the various background components. To subtract the instrumental background components, spectra extracted from the NXB dataset were used, again rescaled to match the observed count rate at high energies (see previous section). Owing to the energy-dependent change of the effective area with off-axis angle, one cannot simply subtract the astrophysical background using a spectrum from a region located at a very different area in the focal plane. Instead, one either has to correct the background spectrum for this effect (see, e.g., Arnaud et al. 2001) or model the background independently (as done by Kaneda et al. 1997). In this work the latter approach was used. Spectra were extracted from three regions:

1. an elliptical region outside of the radio shell and the diffuse excess to model the astrophysical background (the Background region, shown in Fig. 4);

2. an elliptical region encompassing the whole X-ray excess emission (the Full region, shown in Fig. 5);

3. a circular region inside the SNR covering the core of enhanced X-ray emission (the Core region, shown in Fig. 5).

As for the maps of diffuse X-ray emission, all detected point sources were also removed from the event lists for the spectral analysis. The energy-dependent effective area (ARF) and energy response (RMF) files were calculated by averaging over all non-excluded pixels in the respective extraction region, assuming a flat flux distribution. The diffuse Galactic background spectrum was fitted with a two-temperature, non-equilibrium ionization model (2T-NEI), which is known to describe the Galactic diffuse spectrum very well (for studies performed with ASCA and Chandra, see Kaneda et al. 1997; Ebisawa et al. 2005). All model parameters except for the flux normalizations 
Table 2. Extraction regions and spectral fitting results for the diffuse X-ray emission.

\begin{tabular}{lllccccc}
\hline \hline Reg. & RA & Dec & Size $^{a}$ & $\begin{array}{c}\mathrm{Area}^{b} \\
\left(10^{-2} \mathrm{deg}^{2}\right)\end{array}$ & $\Gamma^{c}$ & $\begin{array}{c}\text { Flux }(0.5-10.0 \mathrm{keV})^{d} \\
\left(10^{-13} \mathrm{erg} \mathrm{cm}^{-2} \mathrm{~s}^{-1}\right)\end{array}$ & $\chi^{2} / \mathrm{ndf}^{e}$ \\
\hline Full & $18^{\mathrm{h}} 17^{\mathrm{m}} 59^{\mathrm{s}}$ & $-15^{\circ} 29^{\prime} 2^{\prime \prime}$ & $6 \prime^{\prime} 7,55^{\prime} \cdot 8$ & 2.6 & $-3.8_{+1.0}^{-2.6}$ & $7.0 \pm 3.1$ & 0.89 \\
Core & $18^{\mathrm{h}} 17^{\mathrm{m}} 56^{\mathrm{s}}$ & $-15^{\circ} 27^{\prime} 14^{\prime \prime}$ & $2 ! 7$ & 0.45 & $-2.2_{+0.5}^{-0.5}$ & $3.2 \pm 0.8$ & 1.1 \\
\hline
\end{tabular}

Notes. All quoted uncertainties correspond to the $1 \sigma$ confidence interval; ${ }^{(a)}$ dimensions of the extraction region: semi-major and minor axes for the Full region and radius for the Core region; ${ }^{(b)}$ effective extraction area on the detector (PN), taking excluded point sources, bad pixels/columns, and chip borders into account; ${ }^{(c)}$ photon index of the power-law model; ${ }^{(d)}$ unabsorbed integral flux; ${ }^{(e)}$ value of $\chi^{2}$ divided by the number of degrees of freedom.

of the two thermal components were kept fixed at the bestfit values from (Ebisawa et al. 2005, Table 8). The spectrum from the Background region is described very well by this model $\left(\chi^{2} / \mathrm{ndf}=1.2\right)$ with a total observed surface brightness of $(4.36 \pm 0.14) \times 10^{-7} \mathrm{erg} \mathrm{cm}^{-2} \mathrm{~s}^{-1} \mathrm{sr}^{-1}(0.7-10.0 \mathrm{keV})$. The spectra from the full and the core regions were modeled by the sum of the best-fit 2T-NEI model (rescaled to account for the different extraction areas) and an absorbed power-law component to model the diffuse excess emission. Limited statistics meant that the column density of the absorbed power law was fixed at the total Galactic value in the direction of HESS J1818-154 $\left(1.85 \times 10^{22} \mathrm{~cm}^{-2}\right.$, Dickey \& Lockman 1990). Again, spectra from all three EPIC cameras were fit in parallel.

Table 2 shows the parameters of the extraction regions, as well as the best-fit results of the power-law component for the Full and the Core regions. The quoted errors also take the uncertainties of the background model fit into account, which are, however, very small compared to the statistical uncertainties of the excess emission. As suggested by the different sizes of the emission in the two energy bands, the spectrum from the Core region has a harder spectrum than the Full region. However, this difference in photon index is only marginally significant, mostly due to large uncertainties of the spectral parameters from the Full region arising from its large size and low surface brightness resulting in a lower signal-to-noise ratio. It is worth mentioning that, given the large statistical uncertainties on the spectrum for the Full region, a thermal model is able to describe the data equally well. However, in both cases the model parameters are equally poorly constrained, and therefore the thermal results do not provide much additional information.

\section{Radio observations of $\mathrm{G} \mathbf{1 5 . 4 + 0 . 1}$}

Archival radio data from MAGPIS (White et al. 2005) were used to search for a counterpart to HESS J1818-154 and the newly discovered diffuse X-ray source. VLA observations of the region around $\mathrm{G} 15.4+0.1$ are available at both $20 \mathrm{~cm}$ and $90 \mathrm{~cm}$ wavelengths, where the PSF (here, half-power beam-width) of the observations are $6.2^{\prime \prime} \times 5.4^{\prime \prime}$ and $24^{\prime \prime} \times 18^{\prime \prime}$, respectively. These data are sensitive to structures $<50^{\prime}(90 \mathrm{~cm})$ and $<18^{\prime}(20 \mathrm{~cm})$. In both observations, the SNR has a clear shell structure and appears largely void of emission from the central regions (see Fig. 1). Therefore, since there is no obvious radio counterpart to the X-ray and $\mathrm{TeV}$ source, an upper limit on the flux density from a region corresponding to the Core region (as defined in Sect. 3) was derived. An upper limit on the flux density from the Full region was obtained by scaling the result from the Core region with the ratio of the area between the two regions. This procedure minimizes the contamination of the emission from the shell, while still being conservative, since the most luminous part of the X-ray source is contained inside the Core region. The images were first convolved for a common beam size of $25^{\prime \prime} \times 25^{\prime \prime}$,
Table 3. The $3 \sigma$ flux upper limits from the Core and the Full regions, respectively.

\begin{tabular}{lcc}
\hline \hline Region & $\begin{array}{c}\text { Flux }(90 \mathrm{~cm}) \\
(\mathrm{Jy})\end{array}$ & $\begin{array}{c}\text { Flux }(20 \mathrm{~cm}) \\
(\mathrm{Jy})\end{array}$ \\
\hline Full & $<4.8$ & $<9.6$ \\
Core & $<0.9$ & $<1.8$ \\
\hline
\end{tabular}

and point sources ${ }^{4}$ were removed by masking areas 1.5 times the beam size. The RMS noise was then derived from each (convolved) image and was found to be $\sigma_{90}=20 \mathrm{mJy} \mathrm{beam}^{-1}$ and $\sigma_{20}=4 \mathrm{mJy}$ beam $^{-1}$, respectively. After integrating within the Core and the Full regions, the $3 \sigma$ flux upper limits given in Table 3 were obtained. Uncertainties in the flux density include contributions from i) image noise; ii) calibration; and iii) wrong zero levels, as suggested by Klein et al. (2003).

\section{Discussion}

\subsection{A PWN inside G 15.4+0.1 detected in X-rays and VHE $\gamma$ rays}

The observed X-ray emission exhibits a relatively luminous core component completely contained within the boundaries of the radio shell of G $15.4+0.1$. It is well fit by a hard spectrum power law, which is a clear indication of non-thermal radiation. Surrounding the core component and roughly centered on it, a diffuse X-ray component is observed that fades with distance from the core component. The diffuse emission completely fills the inside of the SNR and partly overlaps with the weaker regions of the radio shell. However, there is no overlap with the northern part of the SNR where the radio emission is strongest. It is therefore very likely that the observed X-ray emission stems from a previously unknown PWN, in which a centrally located pulsar is driving a wind of relativistic leptons that radiates in $\mathrm{X}$-rays via synchrotron mechanism. The discovery of a morphologically compatible $\mathrm{TeV}$ counterpart strengthens this case further, since $\mathrm{TeV} \gamma$ rays can be produced by the same population of leptons through IC scattering on (predominately) cosmic microwave background (CMB) photons. The X-ray and VHE $\gamma$-ray observations of $\mathrm{G} 15.4+0.1$, presented in this paper, therefore show evidence of a PWN located inside the shell of the SNR and thus establish this object as a composite SNR.

X-ray PWNe are generally brighter towards the pulsar thanks to the cooling of the electrons as they propagate outward from the acceleration site. This is also true for G 15.4+0.1, with the difference that the core appears more like a distinct, welldefined region superposed on a much fainter diffuse background. Another possibility is then that the Core region represents the

4 From SIMBAD, http://simbad.u-strasbg.fr/simbad 


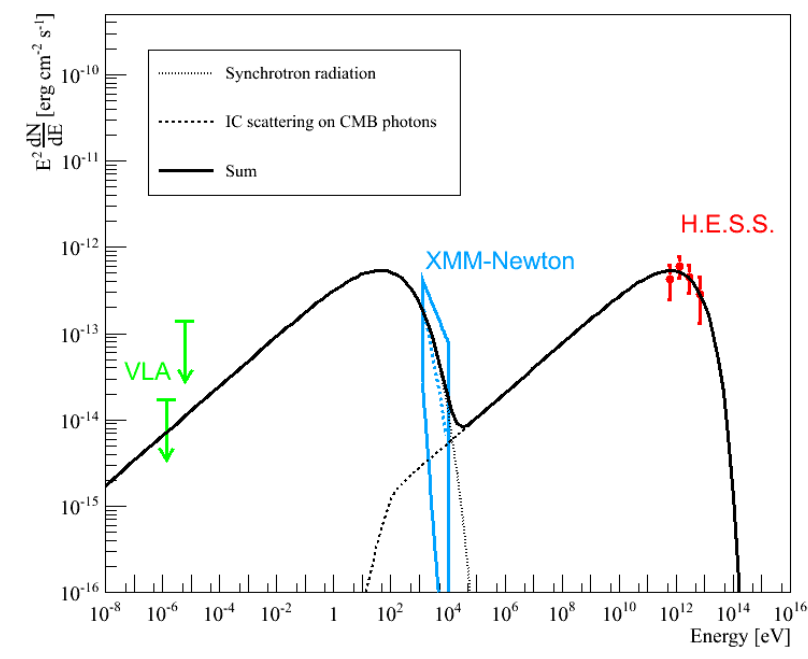

Fig. 6. Spectral energy distribution of the PWN inside G 15.4+0.1 from radio to VHE $\gamma$ rays. The best-fit X-ray spectrum is shown with the $1 \sigma$ error band of the fit. Only statistical errors are shown. Overlaid on the datapoints is the best-fit one-zone leptonic model of the PWN.

termination shock of the pulsar and that its somewhat elongated morphology, observed at high energies, is caused by an anisotropic outflow from the pulsar. However, the extension of the termination shock in PWNe is normally in the range of 0.03-0.3 pc (de Jager \& Djannati-Ataï 2009), which even for a small distance to $\mathrm{G} 15.4+0.1$ of $1 \mathrm{kpc}$, translates to $<1^{\prime}$ and would thus appear point like to XMM-Newton, unlike what is observed in the high-energy X-ray image. The compact feature seen in X-rays is therefore most likely dominated by synchrotron emission from the PWN.

Another possibility is then that the X-ray emission from the Core region is in fact the complete PWN, while the diffuse emission is simply thermal emission from the shell. However, the lack of emission from the bright parts of the radio shell makes it unlikely that this is a dominant part of the emission. It is therefore assumed in the following that the complete observed X-ray emission originates in the cooled wind of the PWN with the caveats noted that part of the emission can come from the pulsar, the termination shock, and from thermal emission from shock-heated material.

Since the extension of the PWN in X-rays and VHE $\gamma$ rays is comparable, the broad-band emission can be assumed to originate in a single population of particles. A one-zone model can therefore be used to model the emission in the context of a PWN scenario. A purely static leptonic model was considered here, where the PWN hosts a population of leptons with a power-law spectrum and an exponential cutoff, inside a region with a uniformly distributed magnetic field. The synchrotron and IC emission from this lepton population were then calculated, only taking IC scattering on CMB photons into account.

Figure 6 shows the broad-band spectral energy distribution (SED) of the newly discovered PWN, together with the best-fit one-zone model to the radio, X-ray, and $\gamma$-ray data overlaid ${ }^{5}$. The radio and X-ray flux points were derived from the Full region. The free parameters of the model are the total energy in relativistic leptons, the spectral index and exponential cutoff energy of the lepton population, and the average magnetic field. The $68 \%$ confidence intervals for the fitted model parameters are given in Table 4. Although the model is very simple, it describes

\footnotetext{
5 The de-absorbed X-ray data points depend on the parameters of the absorption model.
}

Table 4. The $68 \%$ confidence intervals for the parameters of the onezone model in Fig. 6.

\begin{tabular}{lc}
\hline \hline Parameter & Value \\
\hline Magnetic field $(\mu \mathrm{G})$ & $1.5-4.8$ \\
Spectral index $\left(\frac{\mathrm{d} N}{\mathrm{~d} E} \sim E^{-p}\right)$ & $<2.6$ \\
Exp. cutoff $(\mathrm{TeV})$ & $10-80$ \\
Total energy $(\mathrm{erg})$ & $<3 \times 10^{49}$ \\
\hline
\end{tabular}

Notes. One-sided limits are given for the spectral index and total energy since their $\chi^{2}$ contours are highly non-parabolic for lower values than the best-fit value. The total energy is given for a distance of $4.8 \mathrm{kpc}$.

the data well with reasonable physical properties and predicts a low average magnetic field inside the PWN. To better constrain the total energy in the system and the spectral index of the lepton distribution, a detection of the PWN in the radio regime is necessary. A model with a low energy cutoff in the parent lepton distribution, which is expected in the PWN paradigm (see e.g. Wang et al. 2006), has not been considered here.

\subsection{A hadronic scenario for the TeV emission?}

Castelletti et al. (2013) has recently reported the discovery of a molecular cloud in spatial coincidence with the northern part of the SNR shell. The molecular cloud, about $5^{\prime}$ in size, consists of two bright clumps with masses and densities on the order of $10^{3} M_{\odot}$ and $10^{3} \mathrm{~cm}^{-3}$, respectively. From the agreement of the kinematical distances between the SNR and the cloud and from tentative evidence showing that the northern cloud is being disrupted by a strong shock, it was argued that the SNRs interact with the molecular cloud. The authors furthermore show that the observed TeV flux (Hofverberg et al. 2011) can be produced by the decay of $\pi^{0}$ mesons created in the interaction between accelerated protons/nuclei and the molecular cloud, assuming a conversion of a few percent of the supernova explosion energy into relativistic protons.

The molecular cloud extends well outside the shell of the northern boundary of the SNR, and it is therefore clear that the $\mathrm{TeV}$ emission cannot originate in the complete cloud. The southern clump is closer to the center of the SNR, hence also to the $\mathrm{TeV}$ source, and it cannot be ruled out with high confidence that they overlap. A hadronic origin of the $\mathrm{TeV}$ emission is therefore possible, although the lack of emission from the direction of the northern part of the cloud disfavors this scenario.

Also in a hadronic scenario as proposed above, a certain amount of X-ray emission is expected from bremsstrahlung and synchrotron emission from secondary electrons (Gabici et al. 2009). This can, however, not explain the observed X-ray emission observed from $\mathrm{G} 15.4+0.1$ since there is a clear separation between the molecular cloud and the X-ray emission region.

A scenario with a common origin of the $\mathrm{X}$-ray and $\mathrm{TeV}$ emission from a PWN located inside the shell of G $15.4+0.1$ is therefore preferred.

\subsection{Age and evolution of the SNR G 15.4+0.1 and its PWN}

Little is known about the age of the SNR G 15.4+0.1 and its putative pulsar. Castelletti et al. (2013) placed the SNR at a distance of $(4.8 \pm 1.0) \mathrm{kpc}$. At this distance, the angular size of the shell of G $15.4+0.1\left(12^{\prime} \times 18^{\prime}\right)$ corresponds to a physical size of $(17 \times 15)$ pc. From the average physical size of the SNR and assumptions on the ambient medium density and the supernova explosion energy, the Sedov-Taylor model of SNR evolution 
from Blondin et al. (1998) can be used to derive a rough estimate of the age of the SNR. Since the ambient density around $\mathrm{G} 15.4+0.1$ is unknown, a value of $1 \mathrm{~cm}^{-3}$ was adopted, which is valid for a large number of SNRs in the galactic plane. A typical supernova explosion energy of $10^{51} \mathrm{erg}$ was assumed. The model then predicts an age of about 2500 years, placing this SNR in the Sedov phase.

A scenario with a rather young, unevolved system is also supported by the morphology of the SNR and the PWN as seen in radio, X-ray, and TeV energies, assuming the latter is indeed the high-energy counterpart of the PWN. With a X-ray and TeV PWN centered inside the SNR shell, and a roughly symmetric diffuse X-ray component surrounding the inner core, it is unlikely that the reverse shock, which normally hits the expanding PWN after 2000 yrs (van der Swaluw et al. 2001), has had time yet to significantly alter the system.

The age of the putative pulsar can be estimated using the relationship between the $\gamma$-ray to X-ray flux ratio and the characteristic age of the pulsar proposed by Mattana et al. (2009) (and further developed by Kargaltsev et al. 2013). This yields a characteristic age of the pulsar of $17 \mathrm{kyr}$. Interestingly, this suggests an evolved system, in contradiction to the above estimations. However, there are several examples of sources where the true age is significantly less than the characteristic age of the pulsar where the discrepancy is attributed to a fairly constant pulsar spin-down (Roberts et al. 2003; Renaud et al. 2010).

\subsection{Non-detection of a pulsar}

Both the hard galactic (HGAL, see Table 1) X-ray point sources qualify as potential candidate pulsars powering the observed PWN. However, both these sources are located at significant offsets from the peak of the diffuse X-ray emission, which is generally expected to be close to the location of the pulsar, particularly in younger systems. It therefore seems unlikely that these correspond to the pulsar powering this synchrotron nebula.

The non-thermal X-ray emission from a PWN is usually a factor of $\sim 10$ greater than the $\mathrm{X}$-ray emission from its pulsar ( $\mathrm{Li}$ et al. 2008). The expected X-ray flux from the putative pulsar associated to HESS J1818-154 should therefore be on the order of $\left(10^{-14}-10^{-13}\right) \mathrm{erg} \mathrm{cm}^{-2} \mathrm{~s}^{-1}(0.5-10.0 \mathrm{keV})$, which is considerably higher than the point-source sensitivity of the current $X M M-N e w t o n$ observation. A non-detection of a pulsar candidate in this observation would therefore be surprising. However, there are some cases where predominantly thermal X-ray radiation has been detected from pulsars powering young PWNe with black-body temperatures of $\sim 0.1 \mathrm{keV}$ (see, e.g., Hessels et al. 2004). If this was the case for HESS J1818-154, such a source might be challenging to detect, given the high expected absorption column density due to the SNRs large distance.

The VLA observations of G $15.4+0.1$ show no signs of a pulsar either, but in this case it is not surprising given the sensitivity of the observations. A typical young radio pulsar located at $4.8 \mathrm{kpc}$ with an assumed luminosity of $L_{20 \mathrm{~cm}} \sim 2.4 \mathrm{mJy} \mathrm{kpc}^{2}$ (like the median for low-luminosity young rotation powered pulsars as estimated by Camilo et al. 2006) would have a flux density of $S_{20 \mathrm{~cm}} \sim 0.1 \mathrm{mJy}$, considerably lower than the sensitivity of current VLA data. Furthermore, beaming effects could also make the pulsar virtually undetectable in radio.

\section{Conclusions}

A new VHE $\gamma$-ray source, HESS J1818-154, has been discovered toward the direction of the SNR G 15.4+0.1 with a flux above $0.42 \mathrm{TeV}$ of $\left(0.9 \pm 0.3_{\text {stat }} \pm 0.2_{\text {sys }}\right) \times 10^{-12} \mathrm{~cm}^{-2} \mathrm{~s}^{-1}$. The centroid of the source is at, or near, the center of the SNR, and a pure shell-type origin of the emission can be ruled out with high confidence from the observed size of the source. The VHE $\gamma$-ray emission therefore likely originates in a previously unknown source located inside the shell of G 15.4+0.1.

Follow-up observations of HESS J1818-154 with $X M M$-Newton have revealed a diffuse source of X-rays coincident with the $\mathrm{TeV}$ emission. The $\mathrm{X}$-ray emission exhibits a two-component morphology with a hard-spectrum core component surrounded by a fainter halo that fills the SNR cavity completely. The energy flux of the total diffuse X-ray emission is $(7.0 \pm 3.1) \times 10^{-13} \mathrm{erg} \mathrm{cm}^{-2} \mathrm{~s}^{-1}(0.5-10.0 \mathrm{keV})$. The observed VHE $\gamma$-ray emission is morphologically compatible with the extended X-ray source.

The morphological and spectral characteristics of the X-ray emission provide strong evidence of a PWN located inside the shell of G 15.4+0.1. The detection of a morphologically compatible $\mathrm{TeV}$ counterpart to the X-ray PWN further strengthens this scenario. These observations therefore classify this object as a composite SNR. Only a handful of such sources have been detected at $\mathrm{TeV}$ energies, and $\mathrm{G} 15.4+0.1$ is the first case where the composite nature has been discovered first and classified on the basis of VHE $\gamma$-ray observations. A one-zone leptonic model describes the broad-band emission well, predicting a low average magnetic field inside the PWN. Two hard-spectrum point sources discovered in the XMM-Newton observation qualify as potential candidate pulsars powering the observed PWN. However, neither of them constitutes a strong candidate since they are both offset relative to the peak X-ray emission. No pulsar candidates have been discovered in archival radio observations of the SNR.

Castelletti et al. (2013) recently discovered a molecular cloud in spatial coincidence with the northern part of the SNR shell and then suggested a hadronic origin of the TeV emission. Although this scenario cannot be excluded, the so far tentative evidence for an interaction between the MC and the shell, the poor morphological match between the $\mathrm{MC}$ and the $\mathrm{TeV}$ source, and the need to invoke an additional mechanism to explain the $\mathrm{X}$-ray emission makes a hadronic scenario unlikely.

Future deep radio observations are promising to confirm that a powerful pulsar is located at the center G 15.4+0.1. Also, searches for a counterpart to the X-ray synchrotron nebula in radio would allow the injection spectrum of the underlying lepton population to be better constrained. Furthermore, extending the VHE $\gamma$-ray coverage into lower energies would probably facilitate the detection of the IC peak, which is another crucial component for further constraining the parameters of the lepton population. Thus, future observations with H.E.S.S. II and CTA are highly desirable.

Acknowledgements. The support of the Namibian authorities and of the University of Namibia in facilitating the construction and operation of H.E.S.S. is gratefully acknowledged, as is the support by the German Ministry for Education and Research (BMBF), the Max Planck Society, the German Research Foundation (DFG), the French Ministry for Research, the CNRS-IN2P3 and the Astroparticle Interdisciplinary Programme of the CNRS, the UK Science and Technology Facilities Council (STFC), the IPNP of the Charles University, the Czech Science Foundation, the Polish Ministry of Science and Higher Education, the South African Department of Science and Technology and National Research Foundation, and the University of Namibia. We appreciate the excellent work of the technical support staff in Berlin, Durham, Hamburg, Heidelberg, Palaiseau, Paris, Saclay, and in Namibia in the construction and operation of the equipment. This research has made use of software provided by the Chandra X-ray Center (CXC) in the application package CIAO. 


\section{References}

Abramowski, A., Acero, F., Aharonian, F., et al. 2012, A\&A, 548, A46 Aharonian, F., Akhperjanian, A. G., Aye, K.-M., et al. 2005, A\&A, 432, L25 Aharonian, F., Akhperjanian, A. G., Bazer-Bachi, A. R., et al. 2006a, A\&A, 457, 899

Aharonian, F., Akhperjanian, A. G., Bazer-Bachi, A. R., et al. 2006b, Nature, 439,695

Aharonian, F., Akhperjanian, A. G., Bazer-Bachi, A. R., et al. 2006c, A\&A, 460, 365

Arnaud, K. A. 1996, in Astronomical Data Analysis Software and Systems V, ASP Conf. Ser., 101, 17

Arnaud, M., Neumann, D. M., Aghanim, N., et al. 2001, A\&A, 365, L80

Baring, M. G. 1997, in Very High Energy Phenomena in the Universe; Moriond Workshop, eds. Y. Giraud-Heraud, \& J. Tran Thanh van, 97

Berge, D., Funk, S., \& Hinton, J. 2007, A\&A, 466, 1219

Blondin, J. M., Wright, E. B., Borkowski, K. J., \& Reynolds, S. P. 1998, ApJ, 500,342

Blondin, J. M., Chevalier, R. A., \& Frierson, D. M. 2001, ApJ, 563, 806

Brogan, C. L., Gelfand, J. D., Gaensler, B. M., Kassim, N. E., \& Lazio, T. J. W. 2006, ApJL, 639, 25

Camilo, F., Ransom, S. M., Gaensler, B. M., et al. 2006, ApJ, 637, 456

Carrigan, S., Brun, F., Chaves, R. C. G., et al. 2013, in Proceedings of the 48th Rencontres de Moriond

Carter, J. A., \& Read, A. M. 2007, A\&A, 464, 1155

Castelletti, G., Supan, L., Dubner, G., Joshi, B. C., \& Surnis, M. P. 2013, A\&A, 557, L15

de Jager, O. C., \& Djannati-Ataï, A. 2009, in Astrophys. Space Sci. Lib. 357, ed. W. Becker, 451

de Naurois, M., \& Rolland, L. 2009, Astropart. Phys., 32, 231

Dickey, J. M., \& Lockman, F. J. 1990, ARA\&A, 28, 215

Ebisawa, K., Tsujimoto, M., Paizis, A., et al. 2005, ApJ, 635, 214

Eger, P., Domainko, W., \& Clapson, A. 2010, A\&A, 513, A66

Eger, P., Rowell, G., Kawamura, A., et al. 2011, A\&A, 526, A82

Gabici, S., Aharonian, F. A., \& Casanova, S. 2009, MNRAS, 396, 1629

Gaensler, B. M., \& Slane, P. O. 2006, ARA\&A, 44, 17

Gillessen, S., Hinton, J., \& Funk, S. 2005, AIP Conf. Proc., 745, 758

Helfand, D. J., \& Becker, R. H. 1987, ApJ, 314, 203

Hessels, J. W. T., Roberts, M. S. E., Ransom, S. M., et al. 2004, ApJ, 612, 389

Hillas, A. 1985, in International Cosmic Ray Conference, 3, 445

Hofverberg, P., Chaves, R., Mehault, J., \& Naurious, M. 2011, International

Cosmic Ray Conference, 7, 247

Kaneda, H., Makishima, K., Yamauchi, S., et al. 1997, ApJ, 491, 638

Kargaltsev, O., Rangelov, B., \& Pavlov, G. G. 2013 [arXiv: 1305 . 2552]

Klein, U., Mack, K.-H., Gregorini, L., \& Vigotti, M. 2003, A\&A, 406, 579

Li, T., \& Ma, Y. 1983, ApJ, 272, 317

Li, X.-H., Lu, F.-J., \& Li, Z. 2008, ApJ, 682, 1166

Majerowicz, S., Neumann, D. M., \& Reiprich, T. H. 2002, A\&A, 394, 77

Matheson, H., \& Safi-Harb, S. 2005, Adv. Space Res., 35, 1099

Mattana, F., Falanga, M., Götz, D., et al. 2009, ApJ, 694, 12

Nebot Gomez-Moran, A., Motch, C., Barcons, X., et al. 2013, A\&A, 553, A12

Renaud, M., Marandon, V., Gotthelf, E. V., et al. 2010, ApJ, 716, 663

Roberts, M. S. E., Tam, C. R., Kaspi, V. M., et al. 2003, ApJ, 588, 992

Strüder, L., Briel, U., Dennerl, K., et al. 2001, A\&A, 365, L18

Turner, M. J. L., Abbey, A., Arnaud, M., et al. 2001, A\&A, 365, L27

Uchiyama, Y. 2012, in AIP Conf. Ser. 1505, eds. F. A. Aharonian, W. Hofmann, \& F. M. Rieger, 177

van der Swaluw, E., Achterberg, A., Gallant, Y. A., \& Tóth, G. 2001, A\&A, 380, 309

Wang, Q. D., Lu, F. J., \& Gotthelf, E. V. 2006, MNRAS, 367, 937

White, R. L., Becker, R. H., \& Helfand, D. J. 2005, AJ, 130, 586

1 Universität Hamburg, Institut für Experimentalphysik, Luruper Chaussee 149, 22761 Hamburg, Germany

2 Max-Planck-Institut für Kernphysik, PO Box 103980, 69029 Heidelberg, Germany

e-mail: [petter.hofverberg; peter.eger]@mpi-hd.mpg.de

3 Dublin Institute for Advanced Studies, 31 Fitzwilliam Place, Dublin 2, Ireland

4 National Academy of Sciences of the Republic of Armenia, Yerevan

5 Yerevan Physics Institute, 2 Alikhanian Brothers St., 375036 Yerevan, Armenia

6 Institut für Physik, Humboldt-Universität zu Berlin, Newtonstr. 15, 12489 Berlin, Germany

7 Universität Erlangen-Nürnberg, Physikalisches Institut, ErwinRommel-Str. 1, 91058 Erlangen, Germany
8 University of Durham, Department of Physics, South Road, Durham DH1 3LE, UK

9 DESY, 15738 Zeuthen, Germany

10 Institut für Physik und Astronomie, Universität Potsdam, KarlLiebknecht-Strasse 24/25, 14476 Potsdam, Germany

11 Nicolaus Copernicus Astronomical Center, ul. Bartycka 18, 00-716 Warsaw, Poland

12 Department of Physics and Electrical Engineering, Linnaeus University, 35195 Växjö, Sweden

13 Institut für Theoretische Physik, Lehrstuhl IV: Weltraum und Astrophysik, Ruhr-Universität Bochum, 44780 Bochum, Germany

14 Institut für Astro- und Teilchenphysik, Leopold-FranzensUniversität Innsbruck, 6020 Innsbruck, Austria

15 Laboratoire Leprince-Ringuet, École Polytechnique, CNRS/IN2P3, 91128 Palaiseau, France

16 Centre for Space Research, North-West University, 2520 Potchefstroom, South Africa

17 LUTH, Observatoire de Paris, CNRS, Université Paris Diderot, 5 Place Jules Janssen, 92190 Meudon, France

18 LPNHE, Université Pierre et Marie Curie Paris 6, Université Denis Diderot Paris 7, CNRS/IN2P3, 4 Place Jussieu, 75252 Paris Cedex 5, France

19 Institut für Astronomie und Astrophysik, Universität Tübingen, Sand 1, 72076 Tübingen, Germany

20 DSM/Irfu, CEA Saclay, 91191 Gif-Sur-Yvette Cedex, France

21 Astronomical Observatory, The University of Warsaw, Al. Ujazdowskie 4, 00-478 Warsaw, Poland

22 now at Harvard-Smithsonian Center for Astrophysics, 60 garden Street, Cambridge MA, 02138, USA

23 School of Physics, University of the Witwatersrand, 1 Jan Smuts Avenue, Braamfontein, 2050 Johannesburg, South Africa

24 Landessternwarte, Universität Heidelberg, Königstuhl, 69117 Heidelberg, Germany

25 Oskar Klein Centre, Department of Physics, Stockholm University, Albanova University Center, 10691 Stockholm, Sweden

26 Wallenberg Academy Fellow, 75900 Uppsala, Sweden

27 Université Bordeaux 1, CNRS/IN2P3, Centre d'Études Nucléaires de Bordeaux Gradignan, 33175 Gradignan, France

28 Funded by contract ERC-StG-259391 from the European Community

29 University of Namibia, Department of Physics, Private Bag 13301, Windhoek, Namibia

30 School of Chemistry \& Physics, University of Adelaide, 5005 Adelaide, Australia

31 APC, AstroParticule et Cosmologie, Université Paris Diderot, CNRS/IN2P3, CEA/Irfu, Observatoire de Paris, Sorbonne Paris Cité, 10 rue Alice Domon et Léonie Duquet, 75205 Paris Cedex 13, France

32 UJF-Grenoble 1/CNRS-INSU, Institut de Planétologie et d'Astrophysique de Grenoble (IPAG) UMR 5274, 38041 Grenoble, France

33 Department of Physics and Astronomy, The University of Leicester, University Road, Leicester, LE1 7RH, UK

34 Instytut Fizyki Jạdrowej PAN, ul. Radzikowskiego 152, 31-342 Kraków, Poland

35 Laboratoire Univers et Particules de Montpellier, Université Montpellier 2, CNRS/IN2P3, CC 72, Place Eugène Bataillon, 34095 Montpellier Cedex 5, France

36 Laboratoire d'Annecy-le-Vieux de Physique des Particules, Université de Savoie, CNRS/IN2P3, 74941 Annecy-le-Vieux, France

37 Obserwatorium Astronomiczne, Uniwersytet Jagielloński, ul. Orla 171, 30-244 Kraków, Poland

38 Toruń Centre for Astronomy, Nicolaus Copernicus University, ul. Gagarina 11, 87-100 Toruń, Poland

39 Department of Physics, University of the Free State, PO Box 339, 9300 Bloemfontein, South Africa

40 Charles University, Faculty of Mathematics and Physics, Institute of Particle and Nuclear Physics, V Holešovičkách 2, 18000 Prague 8, Czech Republic 